\title{
ESTUDO SOBRE A IMPORTÂNCIA DA CAPACITAÇÃO DO SERVIDOR ADMINISTRATIVO DO HOSPITAL DAS CLÍNICAS DA UNIVERSIDADE FEDERAL DE GOIÁS. ${ }^{1}$
}

\author{
STUDY ON THE IMPORTANCE OF THE SERVER'S ADMINISTRATIVE TRAINING \\ HOSPITAL OF THE FEDERAL UNIVERSITY OF GOIÁS
}

\section{ESTUDIO SOBRE LA IMPORTANCIA DE LA ADMINISTRACIÓN DEL HOSPITAL DE FORMACIÓN DEL SERVIDOR DE LA UNIVERSIDAD FEDERAL DE GOIÁS}

\author{
Marcia Yassunaga Brito \\ Centro de Gestão Empreendedora \\ mybrito@ig.com.br
}

Josmária Lima Ribeiro de Oliveira

FEAD e PUC Minas

josmaria@pucminas.br

\author{
Fabricio Ziviani \\ Universidade FUMEC \\ fazist@hotmail.com \\ Juliana Maria Magalhães Christino \\ FEAD \\ julianam.prof@gmail.com
}

\begin{abstract}
RESUMO
Este trabalho realizou uma pesquisa descritiva quantitativa que teve como campo de pesquisa, o Hospital das Clínicas da Universidade Federal de Goiás. Buscou-se elucidar questões relativas ao processo de capacitação oferecido pela instituição para os servidores administrativos com o interesse de verificar a contribuição da capacitação para a melhoria do desempenho profissional individual e organizacional. Dentre os resultados, verificou-se que na visão dos entrevistados, a melhoria que a capacitação promove no ambiente hospitalar é de crucial importância, trazendo benefícios para os servidores, para a organização e para a sociedade que usufrui desses serviços. Os resultados também apontaram, como sugestões dos participantes da pesquisa, a necessidade de melhoria no próprio processo de capacitação para que o mesmo possa alcançar o seu objetivo principal, a melhoria da produtividade na organização hospitalar.
\end{abstract}

Palavras - Chave: Recursos Humanos, Capacitação, Eficiência.

\begin{abstract}
This study conducted a quantitative descriptive research that had as a research field, the Hospital of the Federal University of Goiás was sought to clarify issues relating to the process of training offered by the institution for administrative servers with interest to verify the contribution of training to improve individual and organizational performance professional. Among the results, it was found that in the view of respondents, improving the training promotes the hospital environment is of crucial importance, bringing benefits to the servers, to the organization and to society that enjoys these services. The results also showed, as suggestions of survey participants, the need to improve the training process itself so that it can achieve its main objective, the improvement of productivity in the hospital organization.
\end{abstract}

KeyWords: Human Resources, Training, Efficiency.

\section{RESUMEN}

En este estudio se realizó una investigación descriptiva cuantitativa que tuvo como campo de investigación, el Hospital de la Universidad Federal de Goiás se trató de aclarar las cuestiones relacionadas con el proceso de

\footnotetext{
${ }^{1}$ Submetido em 29 de Julho de 2013. Aceito em 25 de Setembro de 2013. O artigo foi avaliado segundo o processo de duplo anonimato além de ser avaliado pelo editor. Editores responsáveis: Márcio Augusto Gonçalves e Lucas Maia dos Santos. Reprodução parcial ou total e trabalhos derivativos permitidos com a citação apropriada da fonte.
} 
formación que ofrece la institución para los servidores administrativos de interés para comprobar la contribución de la formación para mejorar el rendimiento profesional individual y organizacional. Entre los resultados, se encontró que, en opinión de los encuestados, la mejora de la formación promueve el ambiente hospitalario es de crucial importancia, aportando beneficios a los servidores, a la organización ya la sociedad que disfruta de estos servicios. Los resultados también mostraron, como sugerencias de los participantes en la encuesta, la necesidad de mejorar el propio proceso de formación para que pueda alcanzar su objetivo principal, la mejora de la productividad en la organización hospitalaria.

Palabras - Clave: Recursos Humanos, Capacitación, Eficiencia.

\section{INTRODUÇÃO}

A globalização trouxe mudanças que levaram as organizações públicas a mudarem seu comportamento, em busca de mais qualidade e eficiência nos seus serviços prestados à população. Na saúde, os usuários, mais conscientes de seus direitos e amparados pela legislação, passam a ser mais exigentes quanto à qualidade e produtividade desses serviços, não se admitindo erros e demora no atendimento. Assim, as organizações hospitalares necessitam aderir às modernas técnicas de gestão para melhor aplicação dos recursos disponíveis e otimizar seus processos produtivos.

Para Mirshawka (1994) o hospital é a mais complexa de todas as empresas modernas, pois tem como função básica a assistência médica curativa, além de constituir-se em centro de educação e de pesquisa de saúde. No mesmo sentido, Drucker (1999) afirma que o hospital é a mais complexa das empresas modernas devido a diversidade das funções, dos serviços e do capital humano, a escassez dos recursos financeiros, a exigência dos clientes, tendo como objetivo prevenir, diagnosticar, restaurar a saúde, formar profissionais e desenvolver pesquisas.

A preocupação dos hospitais, inicialmente, era exclusivamente com a prestação de serviços de saúde, sem dar importância aos processos administrativos. Com a globalização e as mudanças econômicas, o hospital passou a ser visto como empresa, onde todas as atividades, sejam elas fim, meio ou operacional, precisam desempenhar seu papel de forma coordenada e integrada, para evitar que o trabalho seja disperso, desencontrado e não produza os efeitos desejados e não alcance os objetivos estabelecidos, ou seja, a organização e a eficiência não devem existir apenas nas funções ligadas ao paciente, mas abranger também os serviços operacional, de apoio e administrativo, pois se essas funções forem desempenhadas de forma obsoleta, desorganizada e sem métodos, de nada adiantará prover a área técnica com tecnologias, equipamentos e medicamentos de ultima geração. (BORBA, 2010).

A organização hospitalar, como as demais organizações, tem como objetivo produzir resultados positivos a fim de garantir seu funcionamento no presente e no futuro. Para tanto, deve utilizar ferramentas administrativas para revisar resultados, identificar necessidades, corrigir erros e promover, sempre que necessárias, as mudanças para viabilizar uma gestão correta e eficiente (ZOBOLI, 2002).

Segundo Borba (2010), a função administrativa organizacional é a mais importante no trabalho hospitalar, racionalizando e proporcionando lógica na sequência funcional das atividades técnicas administrativas, proporcionado ao profissional que cuida do paciente toda segurança e condições para um atendimento eficiente.

Segundo Marinho (2001), os hospitais universitários, fundados a partir de 1960, por serem hospitais de ensino, além das atividades de assistência à saúde, também desenvolvem atividades de ensino, pesquisa e extensão, tendo um complexo conjunto de atividades: a realização de consultas médicas, exames laboratoriais, diagnósticos clínicos e tratamentos ambulatoriais; o planejamento e a execução de internações e intervenções cirúrgicas; as práticas do ensino e da pesquisa; e o treinamento e formação de profissionais de saúde. 
Considerando que os hospitais universitários atuam em um cenário de recursos escassos e demanda cada vez maior e exigente, necessitam ter processos de compra, faturamento, finanças, controle e fiscalização eficazes, e para tanto precisam de profissionais capacitados e competentes para absorver e enfrentar as mudanças a que estão sujeitos diariamente.

Em virtude do exposto, o problema que se configura como principal nesta pesquisa é a questão: Como a capacitação é utilizada para aprimorar os serviços administrativos desenvolvidos no Hospital das Clínicas da Universidade Federal de Goiás?

\section{REVISÃO DE LITERATURA}

A rapidez das informações, o desenvolvimento tecnológico e a exigência por qualidade e produtividade, tem imposto as organizações grandes desafios, exigindo estratégias e flexibilidade para se adaptarem ao meio em que estão inseridas. Por isso as organizações vêm dando grande destaque às pessoas, visto que as mesmas estão ativamente vinculadas ao processo produtivo e na capacidade de se adaptarem e realizarem as mudanças necessárias (MOREIRA,2004).

Magalhães (2006, p. 12) afirma:

Com a globalização dos negócios, o desenvolvimento tecnológico, o forte impacto das mudanças e o intenso movimento pela qualidade e produtividade, surge uma eloqüente constatação na maioria das organizações: o grande diferencial, a principal vantagem competitiva das empresas decorre das pessoas que nela trabalham.

A evolução na área de recursos humanos trouxe para as empresas o entendimento que são as pessoas o principal recurso para o alcance de seus objetivos. Atualmente, os recursos humanos são considerados pelas organizações como parceiros, que participam na tomada de decisões e contribuem nos resultados organizacionais desejados (GIL, 2001).

Para Moreira (2004), tanto a produtividade quanto a qualidade dependem mais das pessoas do que de sistemas, ferramentas e métodos de trabalho.

\subsubsection{Recursos Humanos na Organização Hospitalar}

Segundo Lacombe (2005), atualmente as organizações, desempenham um papel importante para a sociedade, visto que a maioria dos serviços e produtos é desenvolvida por elas. As pessoas, praticamente por todo período de suas vidas, dependem dos serviços e produtos ofertados pelas empresas: educação, saúde, energia, comunicação, lazer, transporte, e outros. As pessoas não só se beneficiam do que as empresas oferecem, mas também constituem as organizações, sendo essenciais para fazê-las funcionar bem.

Milkovich e Boudreau (2000, p. 19) afirmam:

Ainda que as instalações físicas, os equipamentos e os recursos financeiros sejam necessários para a organização, as pessoas - os recursos humanos - são particularmente importantes. Os recursos humanos trazem o brilho da criatividade para a empresa. As pessoas planejam e produzem os produtos e serviços, controlam a qualidade, vendem os produtos, alocam recursos financeiros e estabelecem objetivos e estratégias para a organização. Sem pessoas eficazes é simplesmente impossível para qualquer empresa atingir seus objetivos.

A organização hospitalar não difere das demais organizações, ou seja, são as pessoas que promovem seu sucesso organizacional. Segundo Bertelli (2004, p. 28): “a valorização e o reconhecimento das pessoas dentro das instituições de saúde são os principais ingredientes para os primeiros passos em direção a uma profunda mudança nos processos e resultados de qualquer organização".

Magalhães (2006), afirma que as transformações no mundo do trabalho, os impactos tecnológicos, a evolução de novas técnicas, materiais, equipamentos, entre outros, demandam 
pessoas qualificadas e atualizadas para acompanhar as inovações que estão presentes em todas as áreas, inclusive a da saúde.

\subsection{Desenvolvimento Humano}

Para Dutra (2009) o desenvolvimento das pessoas é fundamental para a manutenção ou ampliação da competitividade das organizações. Diante de um futuro de complexidade tecnológica e de relações, as pessoas precisam estar preparadas para enfrentar situações cada vez mais complexas e exigentes. Ainda Dutra (2009, p. 101), o desenvolvimento humano está cada vez mais associado à complexidade, definindo o desenvolvimento da pessoa "como a capacidade para assumir atribuições e responsabilidades em níveis crescentes de complexidade".

Bertelli (2004, p. 122) afirma que "o foco da nova geração de sucesso está fundamentado no desenvolvimento das pessoas para a obtenção do desenvolvimento organizacional, treinando, desafiando e estimulando colaboradores a desenvolverem seus potenciais no trabalho e na vida".

Segundo Dutra (2009), para a organização moderna o desenvolvimento de pessoas é primordial para a manutenção de sua competitividade, visto que a mesma, pressionada pelo ambiente externo e pelas pessoas que nelas trabalham, percebe a necessidade de proporcionar o desenvolvimento contínuo de seus trabalhadores, como forma de se manter competitiva, enquanto as pessoas buscam seu desenvolvimento com o objetivo de manter sua empregabilidade.

Reichel (2008, p. 44) afirma:

(...) Quem realmente faz as mudanças organizacionais são as pessoas, e as empresas devem constituir estratégias para criar formas de preparar essas pessoas para conduzir a organização e ultrapassar as instabilidades por meio das mudanças planejadas. $\mathrm{O}$ treinamento muitas vezes é insuficiente para atingir esses objetivos, então faz-se necessário um desenvolvimento de pessoas a médio e longo prazo.

Lacombe (2005) afirma:

A empresa deve procurar desenvolver em seu pessoal múltiplas habilidades que facilitem seu aproveitamento em diferentes posições, pois numa época em que tudo muda com grande velocidade é necessário que as pessoas tenham capacidade para se adaptar a situações diversas.

Para Moreira (2004), o desenvolvimento prepara as pessoas para desempenharem funções mais complexas e diferentes do que estão acostumadas. $O$ desenvolvimento possibilita uma visão mais acurada do futuro individual e organizacional. Desenvolver o potencial das pessoas propicia maior desempenho, aceitação de maiores responsabilidade e comprometimento com os resultados organizacionais.

\subsection{Sobre treinamento e capacitação}

Boog (2007, p.66) afirma que se manter competitivo não é suficiente, é preciso também encontrar novos caminhos e novas soluções para questões inéditas que vem surgindo nos últimos anos. Assim, para que as organizações sejam eficientes, eficazes e sustentáveis nesse ambiente desafiador, é necessário ter pessoas competentes atuando em todas as áreas. $\mathrm{O}$ treinamento é então uma ferramenta eficaz para preparar as pessoas. O autor define treinamento "é o processo para desenvolver e prover conhecimento, habilidades e comportamentos para atender a requisitos". O treinamento melhora o desempenho das ações e tarefas necessárias para atingir os objetivos e resultados desejados. 
Segundo Lacombe (2005), o treinamento aumenta a capacidade do indivíduo para melhor desenvolver suas atividades, podendo também prepará-lo para assumir novas funções, ou seja, o treinamento reflete em mais qualidade e maior produtividade.

Segundo Reichel (2008, p. 26):

Para uma empresa moderna permanecer no mercado enfrentando todas as adversidades no ambiente em que ela vive, deve preocupar-se em estruturar um sistema de treinamento e desenvolvimento de pessoas de uma forma contínua, que valorize e incentive as pessoas a alcançar a excelência com satisfação e qualidade de vida.

Ou seja, quando a organização se preocupa com o aprimoramento da sua força de trabalho, ela está garantindo maior produtividade para a empresa bem como satisfação e realização de seus trabalhadores, pois pessoas bem preparadas significam competitividade para a empresa e empregabilidade para as pessoas.

Marras (2002), afirma que o treinamento tem como objetivos importantes:

- Aumento da produtividade, visto que com a realização de ações de forma correta, haverá economia de tempo, movimentos, insumos, implicando melhor resultado na produção.

- Aumento da qualidade: o conhecimento adquirido ou aperfeiçoado otimiza a qualidade do trabalho.

- Incentivo motivacional: a possibilidade de ser mais eficaz e eficiente motiva o indivíduo a fazer bem feito suas tarefas.

- Otimização pessoal e organizacional: o aumento da produtividade, qualidade, motivação levam a organização à excelência em termos de desenvolvimento organizacional.

- Atendimento de exigências das mudanças: o treinamento facilita e prepara o indivíduo para enfrentar e acompanhar os efeitos gerados pelas mudanças.

\subsection{Desenvolvimento Organizacional}

Segundo Lacombe (2005), o desenvolvimento organizacional surgiu nas décadas de 1960 e 1970, com o objetivo de preparar as organizações para enfrentarem e se adaptarem aos novos mercados e se manterem competitivas em um cenário de constantes mudanças. Preparar e treinar pessoas são estratégias do desenvolvimento organizacional, para que as novas condições do cenário externo sejam enfrentadas dentro de um clima favorável e voltado para a solução de problemas.

Marras (2002, p. 299), define desenvolvimento organizacional como "um processo estratégico de mudança planejada, portanto de longo prazo,que objetiva alavancar a organização a estágios cada vez mais avançados, ao mesmo tempo que integra metas individuais, grupais e empresariais".

Segundo Reichel (2008), o desenvolvimento organizacional está intrinsecamente relacionado com o desenvolvimento das pessoas, pois as mesmas devem ser envolvidas nas mudanças, aprendendo a conviver com as instabilidades, a serem responsáveis por seu autodesenvolvimento, carreira, relacionamentos, e desenvolver a capacidade de aderir às novas tecnologias e formas de gestão.

As constantes mudanças nas áreas políticas, econômicas e sociais exigem, tanto das organizações como das pessoas o preparo antecipado e planejado para enfrentarem os desafios gerados por essas mudanças. Segundo Bergamini (1980, p. 122): “desenvolver organizações é antes de tudo, desenvolver pessoas". Sendo assim, o desenvolvimento organizacional envolve a capacitação como estratégia para o desenvolvimento das organizações. 
Para Sertek (2002), o desenvolvimento organizacional deve propiciar à organização, por meio de seus agentes de transformação, os trabalhadores, o aumento da capacidade de adaptação às novas situações e ambientes. Daí importância de desenvolver as habilidades e conhecimentos de seus funcionários para tornar a organização mais eficiente e eficaz.

Quanto maior e melhor for a participação dos empregados nas decisões e objetivos da empresa, melhores serão seus resultados de produtividade e competitividade. Desenvolver o potencial humano dos empregados torna-se fator decisivo nas políticas de estratégias competitivas (SERTEK, 2002, p. 30).

Segundo Cunha (2005), há uma relação entre desenvolvimento organizacional e treinamento e desenvolvimento humano, já que os mesmos são sistemas básicos para atender as necessidades das organizações e dos indivíduos. A capacitação, que envolve treinamento e desenvolvimento, são fatores importantes numa estratégia de desenvolvimento organizacional. O treinamento possibilita o desenvolvimento da equipe de trabalho, a resolução de problemas, o rendimento com mais eficácia, mudanças de comportamento, desenvolvimento de habilidades e atitudes, itens importantes para o desenvolvimento organizacional.

\subsection{A importância da capacitação}

Com o avanço tecnológico e a competitividade, o sucesso das organizações passaram a depender mais das pessoas do que de outros recursos, tornando imprescindível investir na capacitação de seus trabalhadores, a fim ensiná-los a pensar, desenvolver habilidades e senso crítico, colaborando para a eficiência e produtividade da organização (BERTELLI, 2004).

Segundo Gil (2011, p. 39): "não são apenas as máquinas e os equipamentos que se tornam obsoletos; os conhecimentos e as habilidades também. Mais do que em qualquer outra época, portanto, o trabalhador qualificado é que passa a ser a diferença".

Dentro do conceito de que as organizações dependem das pessoas para produzir, investir em capacitação não é uma escolha, mas uma necessidade fundamental e contínua para que as pessoas possam produzir mais e melhor, caso contrário, podem se tornar menos competitivas e enfrentarem dificuldades para se manterem no mercado (LACOMBE, 2005).

Segundo Boog (2007), cada vez mais as empresas buscam profissionais qualificados e diferenciados, com capacidade de desenvolver novos produtos e serviços. Considerando que o desempenho das empresas está interligado ao dos funcionários, fica claro que o sucesso ou não das empresas depende das pessoas que nelas trabalham.

Se considerarmos que a competitividade da empresa está nas pessoas, nos funcionários que ela emprega, quanto mais preparados e mais competentes eles forem, maiores serão as oportunidades de geração de novas alternativas da vantagem competitiva (BOOG, 2007, p. 327).

No Brasil, segundo Amaral (2008, p. 2), o Estado, em busca de eficiência e de resultados dos serviços prestados, necessita transformar e construir novas práticas de gestão, bem como formar servidores orientados ao mesmo tempo para a eficiência e resultados. Assim, o servidor público deve ter aptidão para lidar com relações complexas e interdependentes, como também com o contexto de escassez. "Precisamos preparar servidores para saber agir e atender demandas sempre crescentes e sem recursos suficientes (humanos, tecnológicos e orçamentários)". Ainda Amaral (2008), para criar valor público, é preciso produzir serviços, resultados e confiança com relação às ações do setor público. Assim, a capacitação é um instrumento estratégico para aumentar a capacidade do governo e proporcionar aos servidores o uso de novas tecnologias e práticas de gestão para obter transparência e eficiência em suas ações. 
Na esfera da Administração Pública Federal brasileira, o processo de capacitação de servidores é regido pelo Decreto $\mathrm{n}^{\circ}$ 5.707, de 23 de fevereiro de 2006, tendo como principais as seguintes finalidades:

I - melhoria da eficiência, e eficácia e qualidade dos serviços públicos prestados ao cidadão;

II - desenvolvimento permanente do servidor público;

III - adequação das competências requeridas dos servidores aos objetivos das instituições, tendo como referência o plano plurianual;

IV - divulgação e gerenciamento das ações de capacitação; e

V - racionalização e efetividade dos gastos com capacitação.

A capacitação contribui para mudar a gestão pública, para inovar e melhorar a capacidade de atender mais e melhorar a qualidade do serviço público (AMARAL, 2008).

Nesse sentido, os hospitais necessitam capacitar seus trabalhadores para se adaptarem ao ambiente de trabalho, e também desenvolverem novos valores, habilidades e competências, tornando-os capazes para enfrentarem os desafios e mudanças do atual mercado, contribuindo para o desenvolvimento organizacional (JERICÓ, 2001).

\section{PROCEDIMENTOS METODOLÓGICOS}

A pesquisa é um estudo de caso, realizado em um hospital universitário do Estado de Goiás, é de cunho quantitativo, traduzindo em números as opiniões e informações que foram classificadas e analisadas, e é descritiva porque descreveu a visão, expectativa e sugestões com relação a importância da capacitação do servidor que atua na área administrativa do Hospital das Clínicas da UFG, para a consecução dos objetivos da instituição.

Os sujeitos de pesquisa são os servidores que desempenham funções administrativas nas áreas administrativa e financeira do Hospital das Clínicas da UFG. A população é constituída por 89 servidores, incluindo servidores com vínculo estatutário e celetista, envolvidos no processo de gestão administrativa do Hospital das Clínicas da UFG. Somente responderam ao instrumento de coleta de dados, 76 servidores, pois o restante encontrava-se em férias e ou período de licença do trabalho.

O questionário aplicado aos servidores é composto de 8 questões de identificação do indivíduo - sem identificar o nome - , 20 questões em uma escala de Likert de 5 pontos que variam de discordo totalmente até concordo totalmente, 3 questões com opção de mais de uma afirmativa, e 1 questão aberta. A pesquisa quantitativa objetivou conhecer através de dados numéricos o comportamento do grupo de entrevistados. O presente trabalho utilizou-se de análises de frequencia para descrever a amostra pesquisada - sexo, idade, escolaridade e situação no trabalho. $\mathrm{Na}$ avaliação da confiabilidade do questionário foi utilizado o índice Alpha de Cronbach, por se tratar de uma escala de concordância e discordância. Os dados foram tabulados utilizando-se do software estatístico SSPS versão 17 e analisados por meio da Análise Fatorial agrupando as variáveis do estudo.

Para melhor analise da pesquisa o questionário foi agrupado em duas variáveis: Desenvolvimento Individual- DI e Desenvolvimento Organizacional-DO.

QUADRO 1: Variáveis da Pesquisa

\begin{tabular}{|c|l|c|c|}
\hline QUESTÃO & \multicolumn{1}{|c|}{ DI } & DO \\
\hline 01 & A capacitação é importante para o meu desempenho profissional. & $\mathrm{X}$ & \\
\hline 02 & $\begin{array}{l}\text { O meu desempenho profissional influencia o processo produtivo total } \\
\text { da instituição em que trabalho. }\end{array}$ & $\mathrm{X}$ \\
\hline 03 & $\begin{array}{l}\text { Os servidores possuem conhecimento das políticas/programas de } \\
\text { capacitação existentes na instituição em que trabalho. }\end{array}$ & $\mathrm{X}$ \\
\hline 04 & As políticas/programas de capacitação existentes na instituição em que & $\mathrm{X}$ & $\mathrm{X}$ \\
\hline
\end{tabular}




\begin{tabular}{|c|c|c|c|}
\hline & $\begin{array}{l}\text { trabalho alcançam o objetivo de aprimorar o meu desempenho } \\
\text { profissional para aumento da produtividade. }\end{array}$ & & \\
\hline 05 & $\begin{array}{l}\text { As políticas/ programas de capacitação disponíveis na instituição em } \\
\text { que trabalho são contínuas e acessíveis. }\end{array}$ & $\mathrm{X}$ & $\mathrm{X}$ \\
\hline 06 & $\begin{array}{l}\text { O processo de capacitação oferecido pela instituição em que trabalho é } \\
\text { suficiente para o meu crescimento individual e profissional. }\end{array}$ & $\mathrm{X}$ & \\
\hline 07 & $\begin{array}{l}\text { Sou estimulado por meus superiores a participar das ações de } \\
\text { capacitação. }\end{array}$ & & $\mathrm{X}$ \\
\hline 08 & Sinto-me motivado a participar dos processos de capacitação. & $\mathrm{X}$ & \\
\hline 09 & $\begin{array}{l}\text { A instituição em que trabalho oferece oportunidades de capacitação aos } \\
\text { Servidores. }\end{array}$ & & $\mathrm{X}$ \\
\hline 10 & $\begin{array}{l}\text { A capacitação oferecida é significativa e compatível com as atividades } \\
\text { exercidas. }\end{array}$ & $\mathrm{X}$ & \\
\hline 11 & $\begin{array}{l}\text { Sou estimulado a aplicar meus conhecimentos adquiridos na } \\
\text { capacitação. }\end{array}$ & $\mathrm{X}$ & \\
\hline 12 & A capacitação influencia na qualidade das minhas tarefas. & $\mathrm{X}$ & \\
\hline 13 & $\begin{array}{l}\text { A capacitação maximiza a minha produtividade na instituição em que } \\
\text { trabalho. }\end{array}$ & & $\mathrm{X}$ \\
\hline 14 & $\begin{array}{l}\text { A capacitação contribui para otimizar mudanças nos processos de } \\
\text { trabalho. }\end{array}$ & & $\mathrm{X}$ \\
\hline 15 & $\begin{array}{l}\text { A capacitação favorece o desenvolvimento de conhecimentos, } \\
\text { habilidades e competências. }\end{array}$ & $\mathrm{X}$ & \\
\hline 16 & $\begin{array}{l}\text { A capacitação proporciona reconhecimento dos colegas e da } \\
\text { instituição. }\end{array}$ & $\mathrm{X}$ & \\
\hline 17 & $\begin{array}{l}\text { A capacitação melhora significativamente os resultados do trabalho da } \\
\text { minha equipe. }\end{array}$ & & $\mathrm{X}$ \\
\hline 18 & $\begin{array}{l}\text { Tenho dificuldades para aplicar em minhas atividades os } \\
\text { conhecimentos adquiridos pela capacitação. }\end{array}$ & $\mathrm{X}$ & \\
\hline 19 & $\begin{array}{l}\text { Sinto-me frustrado e decepcionado quando não posso aplicar em meu } \\
\text { trabalho os conhecimentos adquiridos através da capacitação. }\end{array}$ & $\mathrm{X}$ & \\
\hline 20 & $\begin{array}{l}\text { A instituição em que trabalho avalia os resultados da capacitação no } \\
\text { desempenho individual e organizacional. }\end{array}$ & & $\mathrm{X}$ \\
\hline
\end{tabular}

Fonte: Elaborado pela pesquisadora

Foi utilizado o Alpha de Cronbach para avaliar a confiabilidade de questionário. Esse índice mede a correlação entre as respostas dadas aos itens pelos respondentes. O mesmo segue uma escala de 0 a 1 onde 1 representa uma total confiabilidade de respostas e 0 tende a demonstrar uma certa confusão nas respostas, com cada indivíduo entendendo de forma diferente o questionário.

Foi utilizada também a Análise Fatorial que tem por objetivo agrupar variáveis que possuem semelhanças nas tendências de resposta. Normalmente ela é utilizada para validar blocos de perguntas que já são considerados como parte de uma mesma estrutura. A escolha do número de fatores que farão parte do modelo foi pelo método de observação do gráfico scree-plot (CATTEL, 1966) em que o gráfico analisa algum "salto" de variância total de um valor para outro. O total de fatores é igual a quantidade de autovalores encontrados antes do salto.Para analisar a qual fator cada variável pertence através da matriz ortogonal, foi utilizado o critério Varimax proposto por Kaiser (1958) que em linhas gerais maximiza as correlações das variáveis importantes para o fator e minimizam as que não são importantes.

\subsection{Caracterização da instituição}

O Hospital de Clinicas da Universidade Federal de Goiás-HC/UFG é um órgão suplementar da UFG, vinculado à Reitoria, classificado como Hospital de Ensino da Rede Federal, conforme Portaria N. ${ }^{\circ} 111$ de 23 de março de 1984 do Ministério da Educação. 
O HC/UFG constitui, ainda, um importante formador de conhecimento na área da saúde, por meio do ensino, pesquisa e extensão, tendo contribuído decisivamente para a formação de inúmeros profissionais da área de saúde, como médicos, enfermeiros, nutricionistas, odontologistas, farmacêuticos, psicólogos, entre outros, da UFG e de outras instituições de ensino.

O HC/UFG disponibiliza 316 leitos, realizando aproximadamente por ano, 292.947 consultas, 514.016 exames, 9.327 cirurgias, 2.208 pequenas cirurgias, 667 partos, 10.796 internações e de vários outros procedimentos médicos e hospitalares. Estes números, por si só, já demonstram a dimensão e importância da atuação do HC no contexto do Estado de Goiás.

A estrutura organizacional do hospital, segundo seu regimento interno, é composta pelo Conselho Diretor e Diretoria Executiva, que compreende a Diretoria Geral, Técnica, Administrativa e Financeira, de Enfermagem e de Gestão de Pessoas.

As atividades administrativas estão concentradas especificamente na Diretoria Administrativa/Financeira e de Gestão de Pessoas, abrangendo as principais áreas de Suprimentos, Finanças, administrativa, Manutenção, Desenvolvimento e Gerenciamento de Pessoas.

\section{ANÁLISE E DISCUSSÃO DOS RESULTADOS}

Quando aplicados questionários em escalas de 1 a 5 , onde 1 significa discordo totalmente e 5 concordo totalmente, é necessário avaliar se as respostas dentro de um mesmo contexto (bloco) possuem relação entre si. Espera-se que os indivíduos respondentes compreendam questões de um mesmo bloco em uma mesma "direção" de resposta.

As questões foram analisadas conforme os agrupamentos que fazem parte de um mesmo fator. A Análise Fatorial produziu os seguintes resultados abaixo.

Tabela1: Questões 6, 10 E 15

\begin{tabular}{l|ccccc}
\hline & 1 & 2 & 3 & 4 & 5 \\
\hline $\begin{array}{l}\text { O processo de capacitação oferecido pela instituição em } \\
\text { que trabalho é suficiente para o meu crescimento }\end{array}$ & & & & & \\
individual e profissional & & & & & \\
$\begin{array}{l}\text { A capacitação oferecida é significativa e compatível com } \\
\text { as atividades exercidas }\end{array}$ & 5,3 & 23,7 & 31,6 & 35,5 & 3,9 \\
$\begin{array}{l}\text { A capacitação favorece o desenvolvimento de } \\
\text { conhecimentos, habilidades e competências }\end{array}$ & 2,6 & 3,9 & 3,9 & 63,2 & 26,3 \\
\hline $\begin{array}{l}\text { 1-DISCORDO TOTALMENTE 2-DISCORDO 3-INDIFERENTE 4-CONCORDO 5-CONCORDO TOTALMENTE } \\
\text { Fonte: dados da pesquisa }\end{array}$
\end{tabular}

A questão 6, que diz respeito ao processo de capacitação oferecido pela instituição ser suficiente para o crescimento individual e profissional demonstra que melhorias no processo de capacitação do HC/UFG urgem, tendo em vista o alto índice de discordância. Se a capacitação é direcionada aos servidores e o feedback deles mesmos quanto ao processo de capacitação não é positivo, é preciso procurar a elaboração e implantação de melhorias que atendam a demanda dos servidores quanto ao subsídio para o crescimento individual e profissional. É possível inferir que o objetivo da capacitação de aprimorar o desempenho profissional dos servidores visando a maior produtividade, não está sendo alcançado e se sim, está sendo de forma insuficiente.

Quanto à questão 10, que trata da capacitação significativa e compatível com as atividades exercidas, a maioria dos pesquisados afirmaram que a capacitação, quando ocorre no HC/UFG, quando o servidor tem oportunidade de participar, quando a conhece, tem em si 
significado e está compatível com as atividades exercidas proporcionando a melhoria no desempenho profissional dos servidores, quando aplicados os conhecimentos adquiridos.

A questão 15 tem alto nível de concordância e já expressa o que o funcionário pensa, sobre a capacitação de modo geral, que ela de fato é benéfica. Isso leva a acreditar que o funcionário acredita na utilidade da capacitação, de sua contribuição na ampliação das possibilidades de desenvolvimento de conhecimentos, habilidades e competências.

Tabela 2: Questões 1, 4 e 18

\begin{tabular}{l|ccccc}
\hline & 1 & 2 & 3 & 4 & 5 \\
\hline $\begin{array}{l}\text { A capacitação é importante para o meu desempenho } \\
\text { profissional }\end{array}$ & 2,6 & - & 5,3 & 27,6 & 64,5 \\
$\begin{array}{l}\text { As políticas/programas de capacitação existentes na } \\
\text { instituição em que trabalho alcançam o objetivo de } \\
\text { aprimorar o meu desempenho profissional para aumento } \\
\text { da produtividade }\end{array}$ & 6,6 & 25,0 & 30,3 & 34,2 & 3,9 \\
$\begin{array}{l}\text { Tenho dificuldades para aplicar em minhas atividades os } \\
\text { conhecimentos adquiridos pela capacitação }\end{array}$ & 9,2 & 32,9 & 32,9 & 22,4 & 2,6 \\
$\begin{array}{l}\text { 1-DISCORDO TOTALMENTE 2-DISCORDO 3-INDIFERENTE 4-CONCORDO 5-CONCORDO TOTALMENTE } \\
\text { Fonte: dados da pesquisa }\end{array}$
\end{tabular}

A questão1, que trata da relação da capacitação com o desenvolvimento profissional, demonstra que um número expressivo deles, concorda e concorda totalmente que existe sim uma correlação positiva entre os dois aspectos. Quanto mais exposto à capacitação, melhor é o desempenho profissional do servidor.

A questão 4 verificou a opinião dos participantes sobre o alcance do objetivo da capacitação, qual seja o aprimoramento do desempenho profissional dos servidores para o aumento da produtividade. Sobre esta relação, a maioria dos participantes diz concordar que o aumento da produtividade passa pela melhoria do desempenho profissional dos servidores via participação em programas de capacitação, ou seja, o desempenho profissional de cada servidor é fator de influência sobre o processo produtivo total da instituição.

Quanto à aplicação dos conhecimentos adquiridos no desenvolvimento das atividades, questão 18, observa-se que quando os servidores adquirem o conhecimento por meio da capacitação, conseguem aplicá-los nas atividades cotidianas no HC/UFG, justamente pelo fato de que a capacitação não é desprendida de significado ou de compatibilidade para com a atividade do servidor. Além disso, o servidor é influenciado pela liderança organizacional a usar os conhecimentos adquiridos na capacitação em sua atividade.

Tabela 3: Questão 5

\begin{tabular}{|c|c|c|c|c|c|}
\hline & 1 & 2 & 3 & 4 & 5 \\
\hline $\begin{array}{l}\text { As políticas/ programas de capacitação disponíveis } \\
\text { na instituição em que trabalho são contínuas e } \\
\text { acessíveis }\end{array}$ & 9,2 & 42,1 & 19,7 & 28,9 & - \\
\hline
\end{tabular}

A questão 5 - as políticas/programas de capacitação disponíveis na instituição em que trabalho são contínuas e acessíveis - continua por retratar a ideia de que a instituição não trabalha de forma correta a capacitação pois o funcionário não a visualiza dessa forma.

Tabela 4: Questões 12 e 16 


\begin{tabular}{l|lllll}
\hline & 1 & 2 & 3 & 4 & 5 \\
\hline $\begin{array}{l}\text { A capacitação influencia na qualidade das minhas } \\
\text { tarefas }\end{array}$ & 2,6 & 3,9 & 14,5 & 52,6 & 26,3 \\
$\begin{array}{l}\text { A capacitação proporciona reconhecimento dos } \\
\text { colegas e da instituição }\end{array}$ & 2,6 & 14,5 & 25,0 & 36,8 & 21,1 \\
$\begin{array}{l}\text { 1-DISCORDO TOTALMENTE 2-DISCORDO 3-INDIFERENTE 4-CONCORDO 5-CONCORDO TOTALMENTE } \\
\text { Fonte: dados da pesquisa }\end{array}$
\end{tabular}

As questões 12 e 16 tem alto nível de concordância, mostrando que os funcionários acreditam que são mais reconhecidos quando a fazem porque a qualidade de seu trabalho também sofre uma melhora.

Tabela 5: Questões 11 e 19

\begin{tabular}{l|lllll}
\hline & 1 & 2 & 3 & 4 & 5 \\
\hline $\begin{array}{l}\text { Sou estimulado a aplicar meus conhecimentos } \\
\text { adquiridos na capacitação }\end{array}$ & 6,6 & 14,5 & 34,2 & 38,2 & 6,6 \\
$\begin{array}{l}\text { Sinto-me frustrado e decepcionado quando não } \\
\text { posso aplicar em meu trabalho os conhecimentos }\end{array}$ & 3,9 & 14,5 & 32,9 & 39,5 & 9,2 \\
adquiridos através da capacitação & & & & & \\
$\begin{array}{l}\text { 1-DISCORDO TOTALMENTE 2-DISCORDO 3-INDIFERENTE 4-CONCORDO 5-CONCORDO TOTALMENTE } \\
\text { Fonte: dados da pesquisa }\end{array}$
\end{tabular}

As questões 11 e 19 que tratam da aplicação em suas atividades, por parte dos servidores, dos conhecimentos adquiridos na capacitação estão interligados pelo fato de que o significado positivo da capacitação é ampliado na medida em que supre as necessidades do servidor enquanto realizador de sua tarefa cotidiana no HC-UFG.

Por outro lado, conforme aponta a questão 19, o sentimento que comumente é atrelado ao não uso dos conhecimentos adquiridos na capacitação, é de frustração e isto pode ocorrer quando a capacitação estiver desconectada da verdadeira demanda do servidor que precisa dela para melhorar a execução de suas atividades.

Tabela 6: Questões 3, 4 e 13

\begin{tabular}{l|lllll}
\hline & 1 & 2 & 3 & 4 & 5 \\
\hline $\begin{array}{l}\text { Os servidores possuem conhecimento das } \\
\text { políticas/programas de capacitação existentes na }\end{array}$ & 6,6 & 22,4 & 38,2 & 28,9 & 3,9 \\
instituição em que trabalho & & & & & \\
$\begin{array}{l}\text { As políticas/programas de capacitação existentes } \\
\text { na instituição em que trabalho alcançam o objetivo } \\
\text { de aprimorar o meu desempenho profissional para }\end{array}$ & 6,6 & 25,0 & 30,3 & 34,2 & 3,9 \\
$\begin{array}{l}\text { aumento da produtividade } \\
\begin{array}{l}\text { A capacitação maximiza a minha produtividade na } \\
\text { instituição em que trabalho }\end{array}\end{array}$ & 6,6 & 2,6 & 14,5 & 56,6 & 19,7 \\
\hline $\begin{array}{l}\text { 1-DISCORDO TOTALMENTE 2-DISCORDO 3-INDIFERENTE 4-CONCORDO 5-CONCORDO TOTALMENTE } \\
\text { Fonte: dados da pesquisa }\end{array}$
\end{tabular}

Com relação à questão 3 que procurou verificar acerca do conhecimento por parte dos servidores do HC-UFG das políticas e programas de capacitação existentes na instituição, tem-se que muitos dos participantes são indiferentes $(38,20 \%)$ a esta questão. Essa indiferença deve ser melhor verificada, tendo em vista que a participação dos servidores na capacitação é iniciada a partir do conhecimento da ocorrência da mesma. 
A questão 4 do questionário verificou a opinião dos participantes sobre o alcance do objetivo da capacitação, qual seja o aprimoramento do desempenho profissional dos servidores para o aumento da produtividade. Sobre esta relação, 34,2\% dos participantes dizem concordar e 3,9\% concordam totalmente. $\mathrm{O}$ aumento da produtividade passa pela melhoria do desempenho profissional dos servidores via participação em programas de capacitação. Quando a tarefa é realizada de forma satisfatória, o que inclui a motivação do servidor em aplicar o conhecimento adquirido na capacitação, a finalidade precípua dela é atingida, ou seja, a maximização da produtividade dentro da instituição é algo perceptível. Quando observada a questão 13, é possível notar que a grande maioria dos participantes concorda com essa forma de pensar.

Tabela 7: Questões 2 e 14

\begin{tabular}{l|l|lllll}
\hline & 1 & 2 & 3 & 4 & 5 \\
\hline $\begin{array}{l}\text { O meu desempenho profissional influencia o } \\
\text { processo produtivo total da instituição em que } \\
\text { trabalho }\end{array}$ & & & & & \\
$\begin{array}{l}\text { A capacitação contribui para otimizar mudanças } \\
\text { nos processos de trabalho }\end{array}$ & 2,6 & 3,9 & 9,2 & 51,3 & 32,9 \\
$\begin{array}{l}\text { 1-DISCORDO TOTALMENTE 2-DISCORDO 3-INDIFERENTE 4-CONCORDO 5-CONCORDO TOTALMENTE } \\
\text { Fonte: dados da pesquisa }\end{array}$
\end{tabular}

As questões 2 e 14 mostram que os funcionários acreditam na importância da capacitação, que ela pode mudar a qualidade do trabalho feito e otimizá-lo.Relacionando a capacitação com o desenvolvimento profissional de cada participante, tem-se que um número expressivo deles, concorda e concorda totalmente que existe sim uma correlação positiva entre os dois aspectos. Quanto mais exposto à capacitação, melhor é o desempenho profissional do servidor, segundo a inferência baseada nos dados apresentados acima.

A eficácia e eficiência na execução da atividade que resulta em aumento da produtividade, conquistada por meio da capacitação, por sua vez é derivada das mudanças nos processos de trabalho, de alguma deficiência para a máxima proficiência. A capacitação contribui para otimizar mudanças no processo de trabalho, conforme a assertiva da questão 14, com a qual os participantes responderam concordar e concordarem totalmente em sua maioria.

Tabela 8: Questões 7, 9 e 20

\begin{tabular}{l|ccccc}
\hline & 1 & 2 & 3 & 4 & 5 \\
\hline $\begin{array}{l}\text { Sou estimulado por meus superiores a participar } \\
\text { das ações de capacitação }\end{array}$ & 6,6 & 14,5 & 26,3 & 42,1 & 10,5 \\
$\begin{array}{l}\text { A instituição em que trabalho oferece } \\
\text { oportunidades de capacitação aos servidores }\end{array}$ & 9,2 & 23,7 & 25,0 & 36,8 & 5,3 \\
$\begin{array}{l}\text { A instituição em que trabalho avalia os resultados } \\
\text { da capacitação no desempenho individual e } \\
\text { organizacional }\end{array}$ & 10,5 & 22,4 & 44,7 & 19,7 & 2,6 \\
$\begin{array}{l}\text { 1-DISCORDO TOTALMENTE 2-DISCORDO 3-INDIFERENTE 4-CONCORDO 5-CONCORDO TOTALMENTE } \\
\text { Fonte: dados da pesquisa }\end{array}$
\end{tabular}

Quanto ao encargo e responsabilidade dos gestores e ou superiores na hierarquia organizacional de impulsionar os servidores à participação total na capacitação, a opinião dos participantes, descrita na questão 7 , demonstra um resultado positivo se observado o número de servidores que concordam com tal afirmativa. A influência dos líderes organizacionais é 
um ponto importante que contribui para a efetiva participação dos servidores no processo de capacitação.

Quanto as oportunidades de capacitação ofertadas pela instituição, verificada na questão 9, percebe-se $42,1 \%$ afirmam que sim, são oferecidas as oportunidades de capacitação, porém a maioria opinou por discordar ou se mostrou indiferente. Tais dados demonstram que o alto nível de indiferença para com a questão influencia a ambiguidade de opinião quanto à mesma. Algumas razões podem contribuir para esse resultado, sendo o desconhecimento dos programas de participação, a indisponibilidade do servidor, a desmotivação do servidor, ou ainda o desinteresse pelo item propriamente dito.

Observando a análise da questão 20, é preciso o alerta para com o alto número de indiferença por parte dos participantes com relação à avaliação do desempenho da instituição, isto pode significar o desconhecimento deles da forma pela qual essa avaliação ocorre. O que também chama a atenção é que a maioria discorda de que essa avaliação ocorra no HC/UFG, enquanto existem alguns que concordam. $\mathrm{O}$ que de fato é necessário aqui é que a avaliação seja do conhecimento de todos e devidamente praticada para o acompanhamento dos resultados da capacitação dos servidores do hospital.

Tabela 9: Questão 17

\begin{tabular}{l}
\hline \\
$\begin{array}{l}\text { A capacitação melhora significativamente } \\
\text { resultados do trabalho da minha equipe }\end{array}$
\end{tabular}

Segundo mais de $80 \%$ dos entrevistados, a capacitação melhora significativamente os resultados do trabalho da equipe com a mudança dos processos de trabalho, como o desenvolvimento das habilidades e conhecimentos do servidor, com a melhoria nas relações de trabalho, dentre outros fatores, permitindo que os servidores desempenhem suas funções de modo satisfatório e contributivo para o sucesso organizacional.

Com relação as questões de múltiplas afirmativas, observa-se que todas elas possuem índices acima de $80 \%$, sendo que "aumento de renda", "crescimento profissional dentro da empresa" e "empregabilidade" são itens que tiveram $100 \%$ de concordância de que a capacitação é benéfica para eles.

Como contribuição da capacitação para o desenvolvimento das atividades laborais, os sujeitos participantes da pesquisa entendem que se aumenta a eficiência, totalizando o percentual de $98 \%$ convencidos disso. Já 78\% acredita que amplia-se a segurança no desenvolvimento das tarefas e $90 \%$ afirma que a capacitação melhora a produtividade, além de maximizar a agilidade e melhorar as relações no ambiente de trabalho.

Como contribuição para com o desempenho institucional, a capacitação é vista pelos participantes da pesquisa, como canal que influencia a produtividade (97\%), a eficiência/eficácia (93\%), o aumento nos recursos financeiros (89\%), a redução de custos (74\%), a melhor aplicação dos recursos disponíveis (61\%), a confiabilidade dos serviços prestados $(91 \%)$, a satisfação dos usuários internos e externos (94\%).

Como sugestões para melhoria do processo de capacitação dos servidores administrativos que acontece no $\mathrm{HC} ; \mathrm{UFG}$, feitas pelos participantes da pesquisa, tem-se dentre as principais: maior frequência de cursos de capacitação; realização de capacitação dentro da estrutura física do próprio hospital; disponibilidade de horário; disponibilização de capacitação própria para os servidores de vínculo empregatício celetista; avaliação individual dos servidores; oferta de cursos específicos para atendimento da demanda dos servidores; divulgação das possibilidades de capacitação. 
É possível observar coerência entre a opinião dos participantes e suas sugestões para melhoria do processo de capacitação no HC-UFG.

\section{CONSIDERAÇÕES FINAIS}

Segundo a pesquisa, para os servidores entrevistados, a melhoria que a capacitação promove no ambiente hospitalar é de crucial importância, já que a organização hospitalar possui uma estrutura organizacional complexa, onde a dificuldade de mensurar seu produto, as constantes alterações na legislação, a variabilidade e complexidade do trabalho, os diferentes grupos de profissionais e vínculos empregatícios, as inovações tecnológicas, as cobranças dos órgãos governamentais e da comunidade por um serviço eficiente, exigem profissionais preparados e capacitados, para, dentro desse ambiente tão diverso e complexo, desempenhar suas funções de modo satisfatório e contributivo para a sucesso organizacional.

Por meio da presente pesquisa foi analisada a capacitação oferecida aos servidores públicos do HC-UFG e podem-se inferir, pelos dados da pesquisa, que é conhecido por todos os servidores os benefícios que podem ser originados no processo de capacitação, tanto para o desenvolvimento individual como da organização hospitalar como um todo. Os participantes demonstram o entendimento tal como é preconizado pelo Decreto $n^{\circ} 5707$ de 23 de fevereiro de 2006, referente à Administração Pública Federal, que define a finalidade da capacitação como sendo a melhoria da eficiência/eficácia na atividade do serviço público, assim como a maximização do desenvolvimento das habilidades do servidor público.

Considerando que os resultados da capacitação e a avaliação do desempenho profissional estão interligados, já que os resultados da capacitação somente são conhecidos através da avaliação do desempenho profissional individual e organizacional, o hospital deve procurar avaliar o os resultados da capacitação para verificar se verdadeiramente está ocorrendo melhorias no ambiente de trabalho e promovendo o desenvolvimento dos servidores, para que eles possam enfrentar desafios no trabalho, superando limites e contribuindo para o desenvolvimento organizacional. Considerando que a pesquisa apontou que a maioria discorda de que essa avaliação ocorra no HC-UFG, verifica-se que a avaliação deve ser do conhecimento de todos e devidamente praticada para o acompanhamento dos resultados da capacitação dos servidores do hospital.

Porém questões como a acessibilidade e disponibilidade desta capacitação devem ser observadas de forma mais atenciosa, já que ficou demonstrado que a capacitação não está sendo disponível para todos, evidenciando aparente dificuldade no acesso aos programas de capacitação da instituição. É preciso o alerta que a capacitação é voltada aos servidores e, portanto é necessário que os mesmos tenham acesso irrestrito a ela, isto porque, do contrário não se alcança o objetivo, que é a melhoria do desempenho profissional individual e organizacional.

Além disso, vale dizer que os dados da pesquisa também revelaram a grande utilidade da capacitação enquanto ferramenta para melhoria do desempenho profissional individual e organizacional, e assim sendo é importante que todos os líderes organizacionais estimulem mais os servidores a participarem de cada uma das capacitações, divulgando, motivando e disponibilizando condições necessárias, como tempo, local de realização da capacitação, dentre outras.

Por outro lado, é válido também que sejam formuladas melhores políticas públicas de capacitação a fim de serem implantadas e direcionadas aos servidores do HC-UFG, impulsionando a instituição hospitalar à sua produtividade máxima em qualidade, tendo em vista que é o fator humano no atendimento prestado que realmente produz o diferencial e vantagem enquanto organização. 
Revista de Administração Hospitalar, v.10, n.2, pp. 65-77, maio/agosto, 2013/ Marcia Yassunaga Brito; Fabricio Ziviani; Josmária Lima Ribeiro de Oliveira; Juliana Maria Magalhães Christino.

\section{REFERÊNCIAS}

AMARAL, Helena Kerr. Desenvolvimento de competências de servidores na administração pública brasileira. Revista de Serviço Público - ENAP, nº 57 (out/dez), p. 549-563. Brasília, 2006.Disponível em

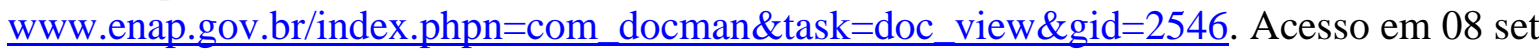
2012.

Capacitação de Servidores. Artigo apresentado no $1^{\circ} \mathrm{Ciclo}$ de Palestras para

Gestores Públicos. Rio Grande do Norte, 2008. Disponível em

www.searh.rn.gov.br/contentproducao/aplicacao/searh_escola/arquivos/pdf/paper_capservido res_helenakerr.pdf. Acesso em 26 out 2012.

BERGAMINI, Cecília Whitacher. Desenvolvimento de Recursos Humanos: uma estratégia de desenvolvimento organizacional. Editora Atlas, São Paulo, 1980.

BERTELLI, Sandra Benevento. Gestão de Pessoas em Administração Hospitalar. Qualitymark Editora, Rio de Janeiro, 2004.

BOOG, G. Gustavo. Manual de treinamento e desenvolvimento: processos e operações. Pearson Education do Brasil, São Paulo, 2007.

BORBA, Valdir Ribeiro, LISBOA, Terezinha Covas. Teoria Geral de Administração Hospitalar: Estrutura e Evolução do Processo de Gestão Hospitalar. Rio de Janeiro: Qualitymark, 2010.

BRASIL. Decreto n. 5.707, de 23 de fevereiro de 2006. Institui a Política e as Diretrizes para o Desenvolvimento de Pessoal da administração pública federal direta, autárquica e fundacional, e regulamenta dispositivos da Lei n. 8.112, de 11 de dezembro de 1990. Diário Oficial da República Federativa do Brasil, Brasília, DF, 24 fev. 2006. Disponível em: <www.planalto.gov.br/legisla.htm>. Acesso em: 25 mai. 2011.

CATTTEL, R. B. The screen test for the number of factors. Multivariate Behavioral Research, v.1, p.140-161, 1966.

CUNHA, Carlos Henrique Lisboa.Mudança Organizacional: Possibilidades e Limites de um Programa de Capacitação e Desenvolvimento Organizacional. Dissertação (Mestrado em Administração Estratégica), Universidade Salvador, Salvador, 2005.

DUTRA, Joel Souza. Gestão por competências: um modelo avançado para o gerenciamento de pessoas. 3.ed. São Paulo: Gente, 2001.

Atlas, 2009.

.Gestão de Pessoas: Modelo, Processos, Tendências e Perspectivas. São Paulo:

DRUCKER, Peter. Desafios gerenciais para o século XXI. São Paulo: Pioneira, 1999.

GIL, Antonio Carlos. Gestão de Pessoas: Enfoque nos papéis profissionais. São Paulo: Atlas, 2011.

JERICÓ, Marli de Carvalho. Análise dos Custos dos Programas de Treinamento e Desenvolvimento de Pessoal de uma Organização Hospitalar. Dissertação (Mestrado em Enfermagem), Universidade de São Paulo.São Paulo, 2001.

KAISER, H.F. The varimax criterion for analytic rotation in factor analysis. Psycometrika, 23, p. 187-200, 1958. 
LACOMBE, Francisco José Masset. Recursos Humanos:Princípios e Tendências. São Paulo Editora Saraiva, 2005.

LAKATOS, Eva Maria; MARCONI , Marina de Andrade. Fundamentos da Metodologia Científica. São Paulo: Atlas, 2001.

MAGALHÃES, Sheilimar Regina Barragão de Sá. Gestão de Recursos Humanos em uma Organização Hospitalar: a utilização de indicadores como ferramentas de gestão. 2006. 105 f. Dissertação (Mestrado Acadêmico de Saúde Pública), Universidade Estadual do Ceará. Fortaleza.

MARINHO, A. Hospitais Universitários: indicadores de utilização e análise de eficiência. Texto de discussão do IPEA. nº33. Rio de Janeiro: IPEA, 2001.

MARRAS, Pierre Jean. Administração de Recursos Humanos: Do operacional ao estratégico. Editora Futura, São Paulo, 2002.

MILKOVICH, George T.; BOUDREAU John W. Administração de Recursos Humanos. São Paulo: Editora Atlas, 2000.

MINGOTI, S. A. Análise de dados através de métodos de estatística multivariada: uma abordagem aplicada. Belo Horizonte: Editora UFMG, 2005.

MIRSHAWKA, Victor. Hospital: Fui bem Atendido, a vez do Brasil. São Paulo: Makron Books, 1994.

MOREIRA, Eunice Aparecida de Lima. Avaliação da Gestão de Capacitação em Órgão Público-Estudo de Caso:Centro Universitário. Dissertação (Mestrado em Sistemas de Gestão), Universidade Federal Fluminense, Niterói, 2004.

REICHEL,Harduin. Treinamento e Desenvolvimento. Curitiba: IESDE Brasil S.A., 2008.

SEVERINO, Antonio Joaquim. Metodologia do Trabalho Científico. 23a ed revista e atualizada. Cortez Editora. São Paulo, 2007.

SERTEK, Paulo. Desenvolvimento Organizacional e Comportamento Ético. Dissertação (Mestrado em Tecnologia), Centro Federal de Educação Tecnológica do Paraná, Curitiba, 2002.

ZOBOLI, Elma L.C.P. Ética e Administração Hospitalar. Editora do Centro Universitário São Camilo. Edições Loyola, São Paulo, 2002. 